Draft version October 8, 2018

Preprint typeset using $\mathrm{LT}_{\mathrm{E}} \mathrm{X}$ style emulateapj v. 5/2/11

\title{
BIG BANG NUCLEOSYNTHESIS AND THE HELIUM ISOTOPE RATIO
}

\author{
RYAN J. CoOKE ${ }^{1,2,3}$ \\ Draft version October 8, 2018
}

\begin{abstract}
The conventional approach to search for departures from the standard model of physics during Big Bang Nucleosynthesis involves a careful, and subtle measurement of the mass fraction of baryons consisting of helium. Recent measurements of this quantity tentatively support new physics beyond the standard model but, historically, this method has suffered from hidden systematic uncertainties. In this letter, I show that a combined measurement of the primordial deuterium abundance and the primordial helium isotope ratio has the potential to provide a complementary and reliable probe of new physics beyond the standard model. Using the recent determination of the primordial deuterium abundance and assuming that the measured pre-solar ${ }^{3} \mathrm{He} /{ }^{4} \mathrm{He}$ meteoritic abundance reflects the primordial value, a bound can be placed on the effective number of neutrino species, $N_{\text {eff }}(\mathrm{BBN})=3.01_{-0.76}^{+0.95}$ (with 95 per cent confidence). Although this value of $N_{\text {eff }}$ supports the standard model, it is presently unclear if the pre-solar ${ }^{3} \mathrm{He} /{ }^{4} \mathrm{He}$ ratio reflects the primordial value. New astrophysical measurements of the helium isotope ratio in near-pristine environments, together with updated calculations and experimental values of several important nuclear reactions (some of which are already being attempted), will lead to much improved limits on possible departures from the standard model. To this end, I describe an analysis strategy to measure the ${ }^{3} \mathrm{He}$ I flux emitted from nearby low metallicity $\mathrm{H}$ in regions. The proposed technique can be attempted with the next generation of large telescopes, and will be easier to realize in metal-poor H II regions with quiescent kinematics.
\end{abstract}

Subject headings: cosmological parameters - galaxies: abundances - methods: numerical — primordial nucleosynthesis

\section{INTRODUCTION}

The standard model of cosmology and particle physics provides our current best physical description of the Universe. Two of the most remarkable predictions of this model, now experimentally confirmed, include the production of the lightest chemical elements during the first minutes after the Big Bang (known as Big Bang Nucleosynthesis, or BBN; Alpher, Bethe, \& Gamow 1948), and the existence of a relic cosmic microwave background $(\mathrm{CMB})$ radiation (Penzias \& Wilson 1965).

Seconds after the Big Bang, ordinary matter consisted mostly of free neutrons and protons. As the Universe expanded and cooled, some neutrons and protons combined to form deuterium (D); soon after, BBN entered full production. Nearly all of the deuterium nuclei were fused to form the more tightly bound helium-4 $\left({ }^{4} \mathrm{He}\right)$ nuclide. Other light nuclides were also produced, but in much lower abundance, including the lighter isotope of helium $\left({ }^{3} \mathrm{He}\right)$ and a very small amount of lithium-7 $\left({ }^{7} \mathrm{Li}\right)$. After $\sim 20$ minutes, the nuclear reactions were quenched by the declining temperature and density of the Universe, and $\mathrm{BBN}$ was complete (for a review, see Steigman 2007, Cyburt et al. 2015).

The relative abundances of the primordial nuclides produced during $\mathrm{BBN}$ are sensitive to the universal expansion rate, and the cosmic density of ordinary matter (i.e. the cosmic baryon density, $\Omega_{\mathrm{B}, 0}$ ). The expansion rate is set by the total energy density of CMB photons and relativistic particles, which for the standard model includes electrons, positrons

\footnotetext{
${ }^{1}$ Department of Astronomy and Astrophysics, University of California, 1156 High Street, Santa Cruz, CA 95064, USA

${ }^{2}$ UCO/Lick Observatory, University of California, Santa Cruz, CA 95064, USA

${ }^{3}$ Hubble Fellow; email: rcooke@ucolick.org
}

and three flavors of neutrino. Typically, the various contributions to the expansion rate are collected and parameterized by an "effective number of neutrino species", $N_{\text {eff }}$, where the standard model value corresponds to $N_{\text {eff }}=3.046$ (Mangano et al. 2005). Deviations from this value could indicate new physics not presently captured by the standard model.

The dependence of each primordial element ratio on $\Omega_{\mathrm{B}, 0}$ and $N_{\text {eff }}$ can be determined from detailed numerical calculations of BBN. Historically, each primordial nuclide is compared relative to hydrogen. For example, the primordial deuterium abundance $(\mathrm{D} / \mathrm{H})$ has commonly been used to estimate the cosmic baryon density (Wagoner, Fowler, \& Hoyle 1967), while the mass fraction of all baryons consisting of ${ }^{4} \mathrm{He}\left(\mathrm{Y}_{\mathrm{P}}\right)$ depends strongly on $N_{\text {eff }}$ and is relatively insensitive to $\Omega_{\mathrm{B}, 0}$ (Steigman, Schramm, \& Gunn 1977).

Similar measurements of $\Omega_{\mathrm{B}, 0}$ and $N_{\mathrm{eff}}$ are also available through a careful analysis of the CMB temperature fluctuations, recently recorded in exquisite detail by the Planck Collaboration et al. (2015). Their analysis provides $\Omega_{\mathrm{B}, 0} h^{2}(\mathrm{CMB})=0.02229_{-0.00040}^{+0.00039}$ and $N_{\mathrm{eff}}(\mathrm{CMB})=3.04 \pm 0.36$ (95 per cent confidence limits), where $h$ is the Hubble constant in units of $100 \mathrm{~km} \mathrm{~s}^{-1} \mathrm{Mpc}^{-1}$. Recent measurements of D/H (Pettini \& Cooke 2012; Cooke et al. 2014) provide an independent bound on $\Omega_{\mathrm{B}, 0} h^{2}(\mathrm{BBN})$ that is in good agreement with the values determined from the $\mathrm{CMB}$. However, the latest measurements of $\mathrm{Y}_{\mathrm{P}}$ suggest tentative, but inconclusive evidence favoring $N_{\text {eff }}(\mathrm{BBN})>N_{\text {eff }}(\mathrm{CMB})$. Specifically, the standard model value of the primordial ${ }^{4} \mathrm{He}$ mass fraction, inferred from the $\mathrm{CMB}$, is $\mathrm{Y}_{\mathrm{P}}=0.24668 \pm 0.00007$ (68 per cent confidence limits; Planck Collaboration et al. 2015). A recent survey conducted by Izotov, Thuan, \& Guseva (2014) found $Y_{P}=0.2551 \pm 0.0022$, which constitutes a $3.8 \sigma$ deviation from the standard model expectation. Using a subset of the Izotov, Thuan, \& Guseva (2014) survey data, Aver, Olive, 
TABLE 1

H ISOTOPE RATIO COEFFICIENTS

\begin{tabular}{ccccc}
\hline \hline$m$ & $n$ & & & \\
\cline { 2 - 5 } & 0 & 1 & 2 & 3 \\
\hline 0 & 29.428 & 3.7763 & -0.18882 & 0.045346 \\
1 & -3490.0 & -437.76 & 38.547 & -8.7506 \\
2 & $1.8127 \times 10^{5}$ & 21897.0 & -2849.5 & 624.51 \\
3 & $-4.4923 \times 10^{6}$ & $-5.1455 \times 10^{5}$ & 90285.0 & -19402.0 \\
4 & $4.3247 \times 10^{7}$ & $4.6528 \times 10^{6}$ & $-1.0411 \times 10^{6}$ & $2.2120 \times 10^{5}$ \\
\hline
\end{tabular}

\& Skillman (2015) derived a value $\mathrm{Y}_{\mathrm{P}}=0.2449 \pm 0.0040$, which is more consistent with the standard model. Thus, the different values of $Y_{P}$ derived by these authors might be due to systematic differences in the analysis strategies adopted.

At present, it is unclear if additional, unaccounted for systematic uncertainties are biasing the best measurements of $\mathrm{Y}_{\mathrm{P}}$, and therefore masquerading as non-standard physics (see e.g. Figure 8 from Steigman 2012). To alleviate this concern, a new, sensitive and reliable probe is required to complement the measurement of $\mathrm{Y}_{\mathrm{P}}$ in the search for possible departures from standard BBN. Until now, the power of combining measurements of the primordial $\mathrm{D} / \mathrm{H}$ and ${ }^{3} \mathrm{He} /{ }^{4} \mathrm{He}$ ratios has not been fully appreciated.

In this letter, I investigate the sensitivity and observational prospects for uncovering new physics beyond the standard model using the $\mathrm{D} / \mathrm{H}$ and ${ }^{3} \mathrm{He} /{ }^{4} \mathrm{He}$ abundance ratios set by $\mathrm{BBN}$. In Section 2, I derive the dependence of these ratios on $\Omega_{\mathrm{B}, 0} h^{2}$ and $N_{\mathrm{eff}}$, and summarize the current best observational determinations. In Section 3 I discuss the importance of obtaining new experimental values of several important reaction cross sections. I also discuss the future potential of measuring the He isotope ratio in near-primordial environments, before highlighting the main conclusions of this work in Section 4

\section{THE BBN ISOTOPES}

The relationships between $\Omega_{\mathrm{B}, 0} h^{2}, N_{\text {eff }}$, and the primordial abundances of $\mathrm{D} / \mathrm{H}$ and ${ }^{3} \mathrm{He} /{ }^{4} \mathrm{He}$ are derived from calculations (Iocco et al. 2009) that use the PArthENoPE code (Pisanti et al. 2008). These results are in good agreement with recent calculations (Cyburt et al. 2015) using the latest determinations of the neutron lifetime (Olive et al.2015) and the reaction rate cross-sections (Xu et al. 2013). The primordial hydrogen and helium isotope number abundance ratios are given by:

$$
y_{\mathrm{H}, \mathrm{He}}=\sum_{n} \sum_{m} a_{n m} \omega_{B}^{n} \Delta N_{\mathrm{eff}}^{m}
$$

where $y_{\mathrm{H}}=10^{5} \times \mathrm{D} / \mathrm{H}, y_{\mathrm{He}}=10^{4} \times{ }^{3} \mathrm{He} /{ }^{4} \mathrm{He}, \omega_{\mathrm{B}}=\Omega_{\mathrm{B}, 0} h^{2}$, and $\Delta N_{\text {eff }}=N_{\text {eff }}-3.046$. The coefficients $a_{n m}$ are provided in Table 1 and Table 2. I adopt a conservative 5\% standard error in $y_{\mathrm{H}}\left(\right.$ Cyburt et al. 2015) and a $3 \%$ standard error in $y_{\mathrm{He}}$ (Steigman 2007) due to uncertainties in the nuclear reaction rates relevant for the $\mathrm{BBN}$ calculations. As discussed further below, this uncertainty dominates the current error budget on $\Omega_{\mathrm{B}, 0} h^{2}(\mathrm{BBN})$ and $N_{\mathrm{eff}}(\mathrm{BBN})$.

The $\mathrm{D} / \mathrm{H}$ number abundance ratio can be determined in near-primordial environments to high precision. The employed technique (Adams 1976) requires a rare, chance alignment between a near-pristine "cloud" of neutral gas and a bright, usually unrelated, background source (typically a quasar). The foreground gas cloud imprints the Lyman series absorption lines of neutral $\mathrm{D}$ and $\mathrm{H}$ on the light from the unrelated background quasar, allowing the relative column density
TABLE 2

HE ISOTOPE RATIO COEFFICIENTS

\begin{tabular}{ccccc}
\hline \hline$m$ & $n$ & & & \\
\cline { 2 - 5 } & 0 & 1 & 2 & 3 \\
\hline 0 & 50.278 & -2.6670 & 0.11296 & 0.11005 \\
1 & -4705.2 & 519.14 & -0.45387 & -30.061 \\
2 & $2.3843 \times 10^{5}$ & -41405.0 & -221.73 & 2564.1 \\
3 & $-5.9383 \times 10^{6}$ & $1.4212 \times 10^{6}$ & 5602.0 & -90887.0 \\
4 & $5.7966 \times 10^{7}$ & $-1.7735 \times 10^{7}$ & 7849.5 & $1.1535 \times 10^{6}$ \\
\hline
\end{tabular}

of $\mathrm{D}_{\mathrm{I}}$ and $\mathrm{H}_{\mathrm{I}}$ to be measured. In this work, I adopt the latest determination $\mathrm{D} / \mathrm{H}=(2.53 \pm 0.04) \times 10^{-5}$ by Cooke et al. (2014), derived from the highest precision measurements currently available.

The helium isotope ratio $\left({ }^{3} \mathrm{He} /{ }^{4} \mathrm{He}\right)$, on the other hand, has received relatively little attention from both the theoretical and observational BBN community. The current best estimate of the $\mathrm{He}$ isotope ratio is measured from the socalled 'quintessence' phase (He-Q) from solar system meteorite samples (Lewis, Srinivasan \& Anders 1975). The isotope ratios of the noble gases that are incorporated into "phase Q' are believed to represent the values that preceded the formation of the solar system, when the Universe was less than 67 per cent of its current age (i.e. less than $\sim 9$ billion years after the Big Bang). Although He-Q may not represent the primordial ${ }^{3} \mathrm{He} /{ }^{4} \mathrm{He}$ (see Section 3.2), this estimate provides an illustrative value that can be used in the present work.

Extracting the noble gases contained in phase $\mathrm{Q}$ requires a two stage process (Busemann, Baur, \& Wieler 2000). In the first step, a meteorite is exposed to hydrochloric and hydrofluoric acid (i.e. demineralization), which leaves a resistant carbonaceous residue. This residue is subsequently exposed to nitric acid, which oxidizes the (presently unknown) carrier ' $Q$ ', thereby releasing the noble gases, including both He isotopes. The ideal meteorites for determining the phase $\mathrm{Q}$ isotopic abundances are those that have the lowest cosmic ray exposure age, since the isotope ratio will be less affected by cosmogenic He production. The best current determination of the He-Q isotope ratio comes from the Isna meteorite (Busemann, Baur, \& Wieler 2000, 2001), which has a remarkably short cosmic ray exposure age, $\mathrm{T}_{21}=150000 \mathrm{yrs}$ (Scherer \& Schultz 2000). The Isna He-Q isotope ratio is ${ }^{3} \mathrm{He} /{ }^{4} \mathrm{He}=(1.23 \pm 0.02) \times 10^{-4}$.

The BBN contours for the above observations and calculations are presented in the left panel of Fig. 1. This figure highlights some of the benefits of using the He isotope ratio to test the standard model; the $\mathrm{D} / \mathrm{H}$ and ${ }^{3} \mathrm{He} /{ }^{4} \mathrm{He}$ abundance ratios offer almost orthogonal bounds on $\Omega_{\mathrm{B}, 0} h^{2}$ and $N_{\text {eff }}$. The determination of $N_{\text {eff }}$ therefore depends almost equally on $\mathrm{D} / \mathrm{H}$ and ${ }^{3} \mathrm{He} /{ }^{4} \mathrm{He}$, unlike the combination of $\mathrm{D} / \mathrm{H}+\mathrm{Y}_{\mathrm{P}}$, where $\mathrm{Y}_{\mathrm{P}}$ drives the determination of $N_{\text {eff }}$. Moreover, $\mathrm{D} / \mathrm{H}$, $\mathrm{Y}_{\mathrm{P}}$, and ${ }^{3} \mathrm{He} /{ }^{4} \mathrm{He}$ all exhibiting a very different dependence on $\Omega_{\mathrm{B}, 0} h^{2}$ and $N_{\mathrm{eff}}$, and their combined measurement will provide a highly complementary approach to identify physics beyond the standard model.

Assuming that the pre-solar ${ }^{3} \mathrm{He} /{ }^{4} \mathrm{He}$ ratio reflects the primordial value, and adopting a conservative uncertainty in the BBN calculations $\left(5 \%\right.$ for $y_{\mathrm{H}}, 3 \%$ for $y_{\mathrm{He}}$ ), the following limits are placed on the baryon density and the effective number of neutrino species: $\Omega_{\mathrm{B}, 0} h^{2}(\mathrm{BBN})=0.0227_{-0.0013}^{+0.0016}$ and $N_{\text {eff }}(\mathrm{BBN})=3.01_{-0.76}^{+0.95}(95$ per cent confidence limits $)$. These results agree remarkably well with the standard model of cosmology and particle physics, and the Planck CMB analysis 

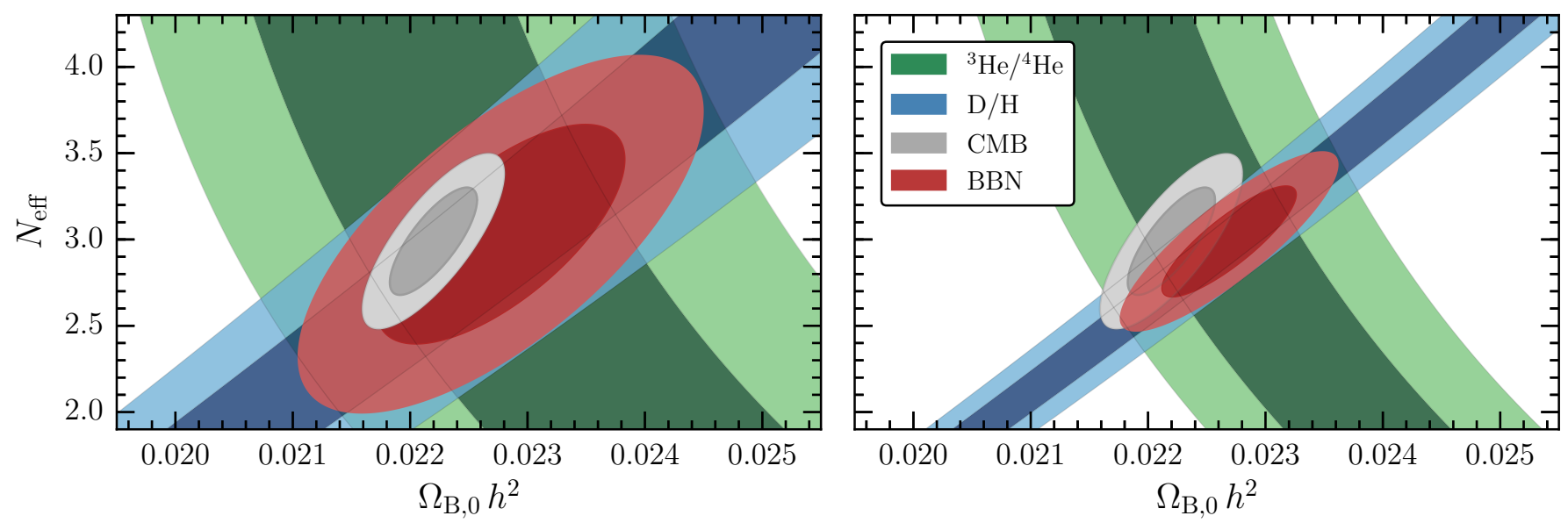

FIG. 1.- Combined confidence contours of the baryon density and the effective number of neutrino species. The D/H (blue) and ${ }^{3} \mathrm{He} /{ }^{4} \mathrm{He}$ (green) contours are displayed, where dark and light shades represent the 68 and 95 per cent confidence contours respectively. Note that the measured ${ }^{3} \mathrm{He} /{ }^{4} \mathrm{He}$ isotope ratio adopted here is derived from solar system meteorite samples, and is intended to be illustrative; this determination of ${ }^{3} \mathrm{He} /{ }^{4} \mathrm{He}$ may not reflect the primordial value. The gray contours illustrate the results from the Planck satellite observations of the CMB temperature fluctuations (Planck Collaboration et al. 2015). The red contours show the combined $\mathrm{D} / \mathrm{H}$ and ${ }^{3} \mathrm{He} /{ }^{4} \mathrm{He}$ (BBN only) confidence regions. The BBN contours in the left panel use a 5\% and 3\% standard error respectively for the $\mathrm{BBN}$ calculations, due to uncertainties in the nuclear reaction rates. The right panel illustrates the same contours, now assuming a $1 \%$ uncertainty on the nuclear reaction rates, and the same observational measures. Note that the $\mathrm{BBN}$ contours in the right panel are comparable in size to the latest $\mathrm{CMB}$ results.

(gray contours in Fig. 11).

\section{FUTURE PROSPECTS}

\subsection{Improving the BBN Reaction Rates}

By improving a few key $\mathrm{BBN}$ nuclear reaction rates (discussed further below), significantly tighter bounds on $\Omega_{\mathrm{B}, 0} h^{2}(\mathrm{BBN})$ and $N_{\mathrm{eff}}(\mathrm{BBN})$ will be possible using observational measures of comparable precision. For example, assuming the same central values for the $\mathrm{BBN}$ reaction rates, a 1 per cent standard error in these rates would reduce the uncertainty on $N_{\text {eff }}(\mathrm{BBN})$ by a factor of 2 . The BBN contours for this level of uncertainty in the reaction rates are illustrated in the right panel of Fig. 11, and are competitive with that of the Planck CMB experiment.

In order for this level of precision to be realized, several $\mathrm{BBN}$ reaction rates must be revisited and measured using modern facilities. The three most uncertain reaction rates that determine the $\mathrm{D} / \mathrm{H}$ abundance are $\mathrm{d}(\mathrm{p}, \gamma)^{3} \mathrm{He}, \mathrm{d}(\mathrm{d}, \mathrm{n})^{3} \mathrm{He}$, and $\mathrm{d}(\mathrm{d}, \mathrm{p})^{3} \mathrm{H}$ (Fiorentini et al. 1998; Nollett \& Burles 2000. Cyburt 2004; Serpico et al. 2004). For the He isotope ratio, the most important reactions are $\mathrm{d}(\mathrm{p}, \gamma)^{3} \mathrm{He}$ and ${ }^{3} \mathrm{He}(\mathrm{d}, \mathrm{p})^{4} \mathrm{He}$. New experimental data for the crucial $d(p, \gamma)^{3} \mathrm{He}$ reaction, which contributes the dominant uncertainty for both the $\mathrm{D} / \mathrm{H}$ and ${ }^{3} \mathrm{He} /{ }^{4} \mathrm{He}$ abundance ratios, are currently being acquired by the Laboratory for Underground Nuclear Astrophysics (LUNA; Broggini et al. 2010; Di Valentino et al. 2014). New data for the remaining reaction rates are now needed.

\subsection{Measuring ${ }^{3} \mathrm{He} /{ }^{4} \mathrm{He}$ in near-pristine environments}

The Isna He-Q measurement currently provides our best indication of the primordial He isotope ratio. However, it remains unclear if $\mathrm{He}-\mathrm{Q}$ is a true reflection of the primordial $\mathrm{BBN}$ value. A measurement of the He isotope ratio in a nearpristine environment, where the post-BBN chemical evolution of ${ }^{3} \mathrm{He}$ is expected to be negligible, will provide a more reliable determination of the truly primordial ${ }^{3} \mathrm{He} /{ }^{4} \mathrm{He}$ ratio. In principle, such a measurement should be possible in known metal-poor $\mathrm{H}_{\text {II }}$ regions by comparing the profiles of two $\mathrm{He}_{\mathrm{I}}$ emission lines, since the isotope shift is different for all $\mathrm{He}_{\mathrm{I}}$ transitions (a relative shift of up to $40 \mathrm{~km} \mathrm{~s}^{-1}$ for the optical and near-infrared emission lines; Morton, Wu, \& Drake
2006). For example, the ${ }^{3} \mathrm{He} 3 \mathrm{D} \rightarrow 2 \mathrm{P}$ singlet transition (He $\mathrm{I}$ 26679) has an isotope shift of $\simeq+22.5 \mathrm{~km} \mathrm{~s}^{-1}$, and the ${ }^{3} \mathrm{He} 2 \mathrm{P} \rightarrow 2 \mathrm{~S}$ triplet transition (He $\mathrm{r} \lambda \lambda 10833$ ) has an isotope shift of $\simeq+35.2 \mathrm{~km} \mathrm{~s}^{-1}$.

The above combination of emission lines is perhaps the best suited to obtain a direct measurement of the ${ }^{3} \mathrm{He}_{\mathrm{I}}$ flux from a metal-poor $\mathrm{H}_{\text {II }}$ region. Assuming a flux ratio of $\mathcal{F}\left({ }^{3} \mathrm{He} /{ }^{4} \mathrm{He}\right)=10^{-4}$, the centroid of the ${ }^{3} \mathrm{He}$ I line would need to be ideally $\gtrsim 5 \sigma$ from the centroid of the ${ }^{4} \mathrm{He}$ I line to ensure that the ${ }^{3} \mathrm{He}$ i emission dominates the signal. For the $1.0833 \mu \mathrm{m}$ He I line, this $\gtrsim 5 \sigma$ shift corresponds to a ${ }^{4} \mathrm{He}$ I profile with a full width at half maximum, FWHM $\lesssim 18 \mathrm{~km} \mathrm{~s}^{-1}$. For comparison, thermal broadening of the ${ }^{4} \mathrm{He}$ line at a temperature of $15,000 \mathrm{~K}$, which is typical of $\mathrm{H}$ II regions (Izotov, Thuan, \& Guseva 2014; Aver, Olive, \& Skillman 2015), would produce a profile with $\mathrm{FWHM} \simeq 13 \mathrm{~km} \mathrm{~s}^{-1}$.

To detect the ${ }^{3} \mathrm{He}$ I feature at $5 \sigma$ confidence, the signalto-noise $(\mathrm{S} / \mathrm{N})$ ratio of the ${ }^{4} \mathrm{He}_{\mathrm{I}}$ feature must exceed $5 \times$ $\sqrt{\mathcal{F}\left({ }^{4} \mathrm{He} /{ }^{3} \mathrm{He}\right)}$, or $\mathrm{S} / \mathrm{N} \gtrsim 500$. This $\mathrm{S} / \mathrm{N}$ requirement would need to be increased if the stellar continuum of the target galaxy is bright, or if the red wing of the ${ }^{4} \mathrm{He}$ I emission line extends over the ${ }^{3} \mathrm{He}$ I emission line. In any case, the primary requirement is that the ${ }^{4} \mathrm{He}$ i profile is smooth and narrow. To confirm that an observed feature is due to ${ }^{3} \mathrm{He}$ i emission, and not a coincident weak ${ }^{4} \mathrm{He}_{\mathrm{I}}$ emission feature, another $\mathrm{He}_{\mathrm{I}}$ emission line with a different isotope shift (e.g. He r 16679) can be inspected. An example comparison of two He i emission lines is presented in Fig. 2 for a Gaussian profile with $\mathrm{FWHM}=13 \mathrm{~km} \mathrm{~s}^{-1}$.

Measuring the ${ }^{3} \mathrm{He}$ I flux from a nearby metal-poor $\mathrm{H}$ iI region might be possible with the next generation of $30+\mathrm{m}$ telescopes. The current instrument concepts of the HIRES and HROS echelle spectrographs, to be mounted on the European Extremely Large Telescope and the Thirty Meter Telescope respectively, are well-designed for this experiment. The wavelength range covered by these instrument concepts includes the best available He I emission lines with a single instrument setup. Moreover, the large telescope aperture greatly facilitates the final $\mathrm{S} / \mathrm{N}$ that can be achieved. However, to realize this measurement, emphasis should now be placed on 

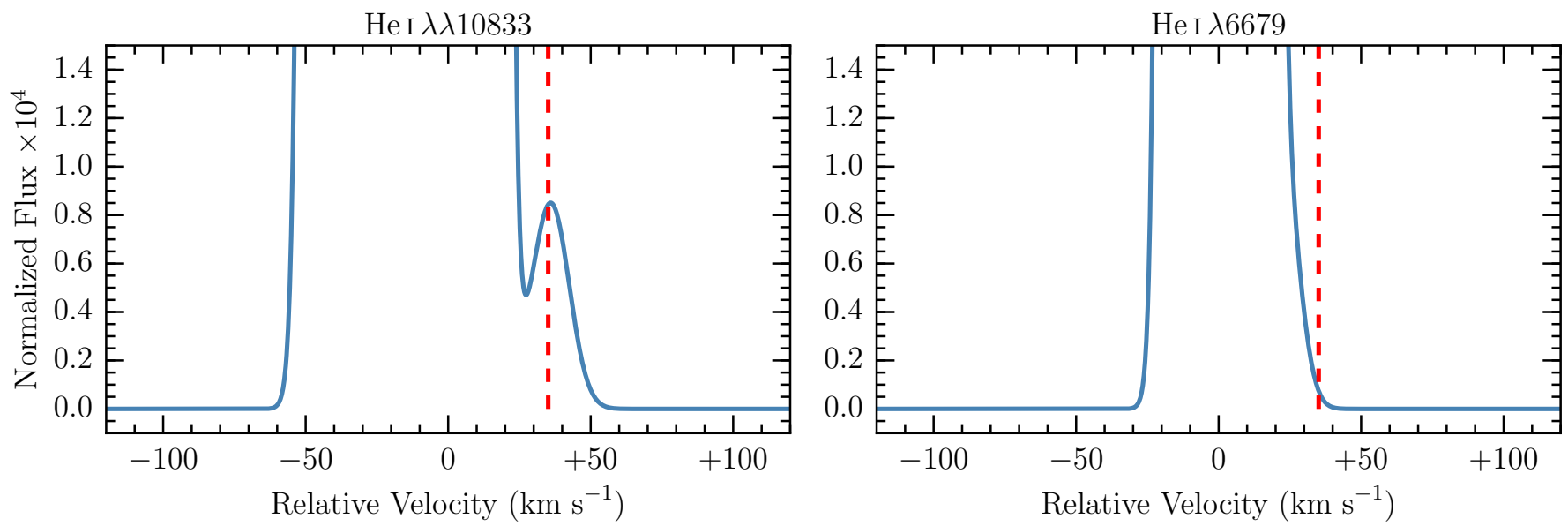

FIG. 2.- Synthetic line profiles for the He I triplet ( $\lambda \lambda 10833$; left panel) and singlet ( $\lambda 6679$; right panel) emission. Both profiles are normalized to the peak flux of the profile. The vertical red dashed lines at a velocity of $+35.2 \mathrm{~km} \mathrm{~s}^{-1}$ in both panels indicate the He isotope shift of the $\lambda \lambda 10833$ line. Note that the He isotope shift of the $\lambda 6679$ line is $+22.5 \mathrm{~km} \mathrm{~s}^{-1}$. Therefore, the detection of an emission feature at $+35.2 \mathrm{~km} \mathrm{~s}^{-1}$ in the $\lambda \lambda 10833$ profile, together with the absence of a feature at the same velocity in the $\lambda 6679$ profile, can be used to confirm the identification as ${ }^{3} \mathrm{He}$ I emission.

discovering suitable low metallicity $\mathrm{H}_{\text {II }}$ regions with quiescent kinematics.

\section{SUMMARY AND CONCLUSIONS}

The primordial abundance of helium-3 $\left({ }^{3} \mathrm{He} / \mathrm{H}\right)$, is generally considered to provide very little constraining power on the physics of $\mathrm{BBN}$, since it is less sensitive to the baryon density than the primordial deuterium abundance, is less affected by the expansion rate than the ${ }^{4} \mathrm{He}$ mass fraction, and a reliable estimate of the primordial value has not been measured. This paper highlights the possibility of combining the deuterium abundance and the helium isotope ratio $\left({ }^{3} \mathrm{He} /{ }^{4} \mathrm{He}\right)$ to simultaneously estimate $\Omega_{\mathrm{B}, 0} h^{2}$ and $N_{\text {eff }}$ at the time of BBN. The following conclusions are drawn:

(i) The $\mathrm{D} / \mathrm{H}$ and ${ }^{3} \mathrm{He} /{ }^{4} \mathrm{He}$ isotope ratios set by $\mathrm{BBN}$ offer almost orthogonal bounds on $\Omega_{\mathrm{B}, 0} h^{2}$ and $N_{\mathrm{eff}}$, unlike $\mathrm{Y}_{\mathrm{P}}$ which is essentially independent of $\Omega_{\mathrm{B}, 0} h^{2}$. Therefore, by combining $\mathrm{D} / \mathrm{H}$ and ${ }^{3} \mathrm{He} /{ }^{4} \mathrm{He}$, evidence for physics beyond the standard model is less influenced by a single measurement.

(ii) If the pre-solar meteoritic value of ${ }^{3} \mathrm{He} /{ }^{4} \mathrm{He}$ reflects the primordial value, the following bounds can be placed on the baryon density, $\Omega_{\mathrm{B}, 0} h^{2}(\mathrm{BBN})=0.0227_{-0.0013}^{+0.0016}$, and the effective number of neutrino species, $N_{\text {eff }}(\mathrm{BBN})=3.01_{-0.76}^{+0.95}$ with 95 per cent confidence, assuming a conservative uncertainty for the measured $\mathrm{BBN}$ reaction rates $\left(5 \%\right.$ for $y_{\mathrm{H}}$ and $3 \%$ for $\left.y_{\mathrm{He}}\right)$.

(iii) In order to achieve bounds on $\Omega_{\mathrm{B}, 0} h^{2}$ and $N_{\text {eff }}$ that are competitive with the latest results from the Planck satellite, several important BBN reaction rates must be redetermined, including $\mathrm{d}(\mathrm{p}, \gamma)^{3} \mathrm{He}, \mathrm{d}(\mathrm{d}, \mathrm{n})^{3} \mathrm{He}, \mathrm{d}(\mathrm{d}, \mathrm{p})^{3} \mathrm{H}$, and ${ }^{3} \mathrm{He}(\mathrm{d}, \mathrm{p})^{4} \mathrm{He}$.

(iv) It is presently unclear to what degree the He-Q isotope ratio is affected by post-BBN nucleosynthesis. It is therefore necessary to obtain a measurement of the He isotope ratio in a near-pristine environment. Nearby metal-poor $\mathrm{H}_{\text {II }}$ regions could be the most promising systems to estimate the primordial ${ }^{3} \mathrm{He} /{ }^{4} \mathrm{He}$ ratio. The He I optical and near-infrared emission lines typically seen in $\mathrm{H}_{\text {II }}$ regions exhibit a variety of isotope shifts (up to $\sim 40 \mathrm{~km} \mathrm{~s}^{-1}$ ), allowing the ${ }^{3} \mathrm{He}$ emission to be unambiguously identified. With a view to this goal, I present a possible strategy to measure the ${ }^{3} \mathrm{He}$ I flux from nearby metal-poor $\mathrm{H}$ in regions.

Although such a delicate measurement will have to wait for the next generation of $30+\mathrm{m}$ telescope facilities, it is critical that the relevant BBN reaction rates are now measured with high precision. Once this goal is achieved, the combined information provided by the primordial $\mathrm{D} / \mathrm{H}$ abundance and the ${ }^{3} \mathrm{He} /{ }^{4} \mathrm{He}$ isotope ratio has the potential to deliver a reliable probe of possible departures from the standard model of physics during the early Universe.

I thank M. Pettini and J. X. Prochaska for useful discussions about the work described in this paper, and for suggesting comments on an earlier draft. I am grateful to the anonymous referee who provided constructive comments that improved the presentation and clarity of this work. R. J. C. is currently supported by NASA through Hubble Fellowship grant HSTHF-51338.001-A, awarded by the Space Telescope Science Institute, which is operated by the Association of Universities for Research in Astronomy, Inc., for NASA, under contract NAS5- 26555. The majority of the analysis and figures presented in this paper were prepared using publicly available РYтноN packages, including Astropy, NumPy, SciPy, Cython, and Matplotlib (Astropy Collaboration et al. 2013; van der Walt, Colbert, \& Varoquaux 2011; Behnel et al.|2011; Hunter 2007).

\section{REFERENCES}

Adams T. F., 1976, A\&A, 50, 461

Alpher R. A., Bethe H., Gamow G., 1948, PhRv, 73, 803

Astropy Collaboration, et al., 2013, A\&A, 558, A33

Aver E., Olive K. A., Skillman E. D., 2015, JCAP, 7, 011

Behnel S., Bradshaw R., Citro C., Dalcin L., Seljebotn D. S., Smith, K., 2011, CSE, 13, 31
Broggini C., Bemmerer D., Guglielmetti A., Menegazzo R., 2010, ARNPS, 60,53

Busemann H., Baur H., Wieler R., 2000, M\&PS, 35, 949

Busemann H., Baur H., Wieler R., 2001, L\&PS, 32, 1598

Cooke R. J., Pettini M., Jorgenson R. A., Murphy M. T., Steidel C. C., 2014, ApJ, 781, 31

Cyburt R. H., 2004, PhRvD, 70, 023505 
Cyburt R. H., Fields B. D., Olive K. A., Yeh T.-H., 2015, arXiv, arXiv:1505.01076

Di Valentino E., Gustavino C., Lesgourgues J., Mangano G., Melchiorri A., Miele G., Pisanti O., 2014, PhRvD, 90, 023543

Fiorentini G., Lisi E., Sarkar S., Villante F. L., 1998, PhRvD, 58, 063506

Hunter, J. D., 2007, CSE, 9, 90

Iocco F., Mangano G., Miele G., Pisanti O., Serpico P. D., 2009, PhR, 472, 1

Izotov Y. I., Thuan T. X., Guseva N. G., 2014, MNRAS, 445, 778

Lewis R. S., Srinivasan B., Anders E., 1975, Science, 190, 1251

Mangano G., Miele G., Pastor S., Pinto T., Pisanti O., Serpico P. D., 2005, $\mathrm{NuPhB}, 729,221$

Morton D. C., Wu Q. X., Drake G. W. F., 2006, CaJPh, 84, 83

Nollett K. M., Burles S., 2000, PhRvD, 61, 123505

Olive K. A., et al. [Particle Data Group Collaboration], 2015, Chin. Phys. C, 38,090001
Penzias A. A., Wilson R. W., 1965, ApJ, 142, 419

Pettini M., Cooke R., 2012, MNRAS, 425, 2477

Pisanti O., Cirillo A., Esposito S., Iocco F., Mangano G., Miele G., Serpico P. D., 2008, Comput. Phys. Commun., 178, 956

Planck Collaboration, et al., 2015, arXiv, arXiv:1502.01589

Scherer P., Schultz L., 2000, M\&PS, 35, 145

Serpico P. D., Esposito S., Iocco F., Mangano G., Miele G., Pisanti O., 2004, JCAP, 12, 010

Steigman G., Schramm D. N., Gunn J. E., 1977, Phys. Lett. B, 66, 202

Steigman G., 2007, ARNPS, 57, 463

Steigman G., 2012, Adv. In High Energy Phys., 2012, 268321

van der Walt S., Colbert S. C., Varoquaux G., 2011, CSE, 13, 22

Wagoner R. V., Fowler W. A., Hoyle F., 1967, ApJ, 148, 3

Xu Y., Takahashi K., Goriely S., Arnould M., Ohta M., Utsunomiya H., 2013, NuPhA, 918, 61 